\title{
Correction to: Assuring Intelligent Ambient Assisted Living Solutions by Statistical Model Checking
}

Ashalatha Kunnappilly, Raluca Marinescu, and Cristina Seceleanu

\section{Correction to:}

Chapter "Assuring Intelligent Ambient Assisted Living

Solutions by Statistical Model Checking" in:

T. Margaria and B. Steffen (Eds.): Leveraging Applications

of Formal Methods, Verification and Validation, LNCS 11245, https://doi.org/10.1007/978-3-030-03421-4_29

In the original version of this chapter, Fig. 3 was incorrect. This has now been corrected. 